\title{
Bounds on dissipation in magnetohydrodynamic problems in plane shear geometry
}

\author{
F. Pétrélis ${ }^{a)}$ \\ Laboratoire de Physique Statistique, ENS, 24 rue Lhomond, 75005 Paris, France \\ A. Alexakis \\ Department of Physics, University of Chicago, Chicago, Illinois 60637 \\ Charles R. Doering \\ Department of Mathematics, University of Michigan, Ann Arbor, Michigan 48109-1109 \\ and Michigan Center for Theoretical Physics, Ann Arbor, Michigan 48109-1120 \\ P. J. Morrison ${ }^{\text {b) }}$ \\ Department of Physics and Institute for Fusion Studies, The University of Texas at Austin, \\ Austin, Texas 78712
}

(Received 3 April 2003; accepted 4 June 2003)

\begin{abstract}
The total dissipation rate for magnetohydrodynamic (MHD) flows in plane geometry with both velocity and magnetic shear is studied. For some boundary conditions it is shown that the lower bound on the dissipation rate is achieved by the equivalent of Stokes flow for MHD. Using the background method [Doering and Constantin, Phys. Rev. Lett. 69, 1648 (1992)] upper bounds for the dissipation rate are calculated. For a shear layer, with both velocity and magnetic shear, parameter dependence of the upper bound is obtained. As a by-product of this calculation, an energy stability domain is calculated. A sheet pinch is also studied, and it is shown that the upper bound tends to zero as the resistivity tends to zero. Thus, an antiturbulence result is obtained. (C) 2003
\end{abstract} American Institute of Physics. [DOI: 10.1063/1.1595649]

\section{INTRODUCTION}

The most prevalent theoretical means for explaining fluid and plasma turbulence is to pursue a statistical description that strives to determine averaged quantities by means of averaging the equations of motion. This leads to the problem of closure (see, e.g., Refs. 1 and 2), which has instigated various physically motivated but ultimately ad hoc assumptions. Alternatively, there is a long history of phenomenological modelling (see, e.g., Sec. 38 of Refs. 3 and 4) and the adoption of other more or less heuristic simplifying assumptions (see, e.g., Refs. 5-10) primarily for the purpose of calculational tractability.

An alternative to the above approaches is to proceed by carefully manipulating the equations of motion according to mathematically justifiable operations, with the goal of obtaining bounds on physical quantities such as the energy dissipation rate. This approach, which makes no ad hoc assumptions but has more modest goals, was pioneered for driven fluid turbulence in the works of Malkus, Howard, and Busse (see, e.g., Refs. 11-13), and has gone by the names of upper bound or optimum theory. Since this early research, upper bound theory has been explored in a variety contexts including that of magnetohydrodynamics (MHD), the subject of the present paper. In Ref. 14 an upper bound was obtained for the ohmic dissipation rate in a turbulent thermal layer permeated by a horizontal magnetic field maintained by dy-

\footnotetext{
${ }^{a)}$ Electronic mail: petrelis@1ps.ens.fr

${ }^{b)}$ Electronic mail: morrison@physics.utexas.edu
}

namo action, in Refs. 15-17 it was applied to an MHD description of the reversed-field pinch, and recently in Ref. 18 it was applied to a sheet pinch configuration.

An allied alternative to upper bound theory, the background method, was developed in the context of fluid turbulence in Ref. 19. This method provides an easier means for obtaining upper bounds by using a mathematical device introduced by Hopf in Ref. 20, whereby one manipulates the equations of motion relative to an assumed background state. This procedure is somewhat akin to the early "thermodynamic approach" of plasma physics for finding upper bounds on instability growth rates (see, e.g., Refs. 21 and 22). Subsequent to Ref. 19 the background method has been explored in a variety of contexts (e.g., Refs. 23-26). The purpose of the present paper is to explore the background method in the context of incompressible MHD, and this study is continued in a companion paper (see Ref. 27). Here we describe the method, discuss boundary conditions, and apply the method to field aligned flows, while in Ref. 27 we apply it to cross field or Hartmann flows, which provides the possibility of direct comparison with liquid metal experiments.

We focus on the total MHD dissipation rate, the sum of the viscous and Ohmic dissipation rates integrated over the volume. This global quantity is clearly important, because its time average is equal to the time average of the injected power, which is in turn equal to the heat production in the system. Moreover, the total dissipation rate is expected to be a measure of the temperature increase in the system, which is obviously desirable in the context of fusion experiments, but it is undesirable in the context of MHD dynamo experiments 
in liquid metals, because it tends to result in an increase in the critical value of the velocity needed for dynamo action.

The viscous dissipation rate has been widely studied in hydrodynamics with a history dating to Stokes (see, e.g., Refs. 28 and 29). Stokes' results were improved upon in Ref. 29 , where it was shown that the solution of the Stokes equations minimizes the dissipation rate over all divergence-free fields satisfying the boundary conditions of fixed velocity at the boundary. This Stokes solution is only a solution of the Navier-Stokes equations in the limit of zero Reynolds number, but in most cases a related steady laminar solution exists upon increasing the Reynolds number. However, this solution eventually becomes unstable for sufficiently large Reynolds numbers. From the evolution equation one finds that there is a critical value of the Reynolds number below which dissipation causes a positive definite energy-like measure of any perturbation to decrease in time. This value defines the domain of absolute stability in which the laminar solution is energy stable for all initial conditions (see, e.g., Refs. 28 and 31). For Reynolds numbers outside this domain, the solution can be unstable and the flow can be turbulent.

For such large Reynolds numbers it is impossible to explicitly calculate the solution of the Navier-Stokes equation or even the average value of the dissipation rate. To surmount this difficulty the upper bound and background theories were developed. Howard, Busse, Doering, and Constantin (see, e.g., Refs. 12, 13, and 19) all sought upper bounds for the dissipation rate using a variational formulation with added constraints. Prior to their investigations, Malkus (see Ref. 11) postulated the physical principal that a turbulent fluid actually achieves this upper bound; it is quite striking that the optimizing fields obtained seem to share properties with the time average of the fields measured in experiments.

The main accomplishment of the present work is to obtain explicit upper bounds on the total dissipation rate in MHD flows. However, we also obtain some lower bounds on the dissipation rate. In Sec. II we show that a Stokes-type solution of the MHD equations minimizes the total dissipation over all divergence-free fields satisfying the boundary conditions of fixed velocity and magnetic fields, and therefore this solution provides a lower bound.

The total MHD dissipation rate has been previously studied. Montgomery (e.g., Ref. 30) has argued that driven MHD flows relax to states for which the dissipation rate is minimized subject to constraints such as the constancy of magnetic flux. This is an argument that attempts to explain a universal feature of the dynamics. Because of the constraints imposed, this principal differs from our calculations of Sec. II, which akin to Stokes only imposes the divergence-free conditions.

Next, we use the background method to obtain upper bounds on the dissipation rate for two MHD problems. In Sec. III we consider a layer with both magnetic and velocity shear. Both lower and upper bounds for the dissipation are derived, and their dependence on the parameters of the system are presented. Few MHD energy stability results (in the spirit of Ref. 28) exist, an exception being the results of Ref. 32 (see also Ref. 33). In some cases the energy domain is infinite, which ensures stability of the laminar solution for all values of the control parameter as given in Ref. 34. A byproduct of our dissipation calculations of Sec. III is an improvement on the domain of stability of these previous results.

In Sec. IV we study a model of the plane sheet pinch. Using the background method, we prove that the upper bound for the dissipation tends to zero with resistivity, while all other parameters are held fixed. This can be interpreted as an antiturbulence theorem because there is no residual dissipation in the limit of zero resistivity. A similar result was recently derived for horizontal convection in Ref. 35, but the present calculation shows that the background method allows one to prove such a result. Similar results were obtained contemporaneously in Ref. 36, where the background method was applied to horizontal convection. For horizontal convection at high Rayleigh numbers, the flow is not stationary and develops boundary layers even if the dissipation tends to zero. According to numerical simulations given in Ref. 37, this is not the case for the driven plane sheet pinch in which the static solution is stable. Our result does not prove energy stability, but is consistent with it because the dissipation of the static solution tends to zero with resistivity.

In Sec. $\mathrm{V}$ we summarize and then briefly mention additional work on MHD, namely, that of our companion paper (see Ref. 27) and other possible research.

\section{STOKES-TYPE SOLUTIONS AND LOWER BOUND}

The incompressible MHD equations for the velocity field, $\mathbf{v}$, and magnetic field, $\mathbf{B}$, are the following:

$$
\begin{aligned}
& \frac{\partial \mathbf{v}}{\partial t}=-\mathbf{v} \cdot \nabla \mathbf{v}-\nabla P+\mathbf{B} \cdot \nabla \mathbf{B}+\nu \nabla^{2} \mathbf{v}+\mathbf{f}, \\
& \frac{\partial \mathbf{B}}{\partial t}=-\mathbf{v} \cdot \nabla \mathbf{B}+\mathbf{B} \cdot \nabla \mathbf{v}+\eta \nabla^{2} \mathbf{B}
\end{aligned}
$$

which are to be solved subject to the constraints $\nabla \cdot \mathbf{v}=0$ and $\nabla \cdot \mathbf{B}=0$. Here $\mathbf{f}$ is an applied body force, $P=p / \rho$ $+\mathbf{B}^{2} /(2 \rho)$, where $p$ is the hydrodynamic pressure, $\rho$ the constant density, and $\mathbf{B}^{2} / 2$ is the magnetic pressure. The kinematic viscosity of the fluid is denoted by $\nu$ and the resistivity by $\eta=\left(\mu_{0} \sigma\right)^{-1}$, where $\sigma$ is the conductivity. We consider these equations in a three-dimensional domain $D$.

For the calculations we present, it is essential to be precise about boundary conditions. Choices used in the past have been based on both physics and expediency, and we briefly discuss some of them. The correct physical boundary conditions for MHD are uncertain because any real plasma experiment is bounded by nonideal conductors of some form, along with the existence of insulating gaps that allow for flux penetration, and as one approaches the boundary there may be a region with no plasma or one for which the MHD fluid model is inaccurate. Similar comments apply for liquid metal experiments. Consequently, compromises are made, and there are several choices available that maintain mathematical well-posedness. (See, e.g., Ref. 30 for additional discussion about boundary conditions.)

For a fluid in contact with a solid wall the most natural boundary condition on the velocity field is the no-slip con- 
dition at the boundary, $\partial D:\left.\mathbf{v}\right|_{\partial D}=\mathbf{v}_{b}$, where $\mathbf{v}_{b}$ is the velocity of the boundary. Here we adopt this no-slip choice, but we point out that the stress-free condition, which in twodimensions amounts to fixing the vorticity on $\partial D$, has also been widely used. The stress-free condition is physically more difficult to justify, but can make for easier numerical implementation. Similarly, the vanishing of the normal component of the vorticity has been used in a sequence of papers by Montgomery and collaborators (see, e.g., Ref. 38).

For the magnetic field, a common choice is to suppose that the solid wall is a perfect conductor, in which the magnetic field is frozen. Because $\nabla \cdot \mathbf{B}=0$ implies continuity of the normal component of the magnetic field at an interface, its value is then fixed at the boundary. This condition is naturally refereed to as the "flux-tied" condition because locally the flux through the boundary is preserved. By imposing the surface current in the boundary, one can fix the value of the discontinuity in the tangential component of the magnetic field and, because its value is fixed in the wall, the tangential component of the magnetic field is also fixed at the boundary. This condition, which is properly referred to as the "line-tied" boundary condition, is more difficult to maintain in experiments because, even though the total current can be controlled, local surface currents are induced by the plasma or liquid metal. Nonetheless, this condition was used in early numerical tearing mode calculations (see, e.g., Ref. 39) and we adopt it in the remainder of the present section and in Sec. III. An oft-used alternative to this condition is to fix the tangential component of the electric field at the boundary and then to appeal to Ohm's law to obtain a condition on the tangential plasma current density at the boundary. To facilitate calculation it is often assumed that the resistivity near the boundary is independent of position, which is also difficult to justify. Because this condition involves derivatives of the magnetic field, it can be viewed as the magnetic analog of the fluid stress-free condition. We adopt a version of this condition in our calculations of Sec. IV. Intuitively one expects this condition to result in dynamics that is less rigid near the boundary than line tying.

Although the main object of our consideration is the total energy dissipation rate per unit mass,

$$
\mathcal{D}=\nu\left\langle|\nabla \times \mathbf{v}|^{2}\right\rangle+\eta\left\langle|\nabla \times \mathbf{B}|^{2}\right\rangle,
$$

where $\langle f\rangle=\int_{D} f(x, y, z) d x d y d z / V$ and $V$ is the volume of the domain $D$ that contains the fluid, for convenience in the present section we follow Keller et al. (see, e.g., Ref. 29) and introduce the excess dissipation rate, the dissipation minus twice the power input by the external body force, $\mathcal{D}_{e}:=\mathcal{D}$ $-2\langle\mathbf{f} \cdot \mathbf{v}\rangle$. This quantity obviously reduces to the total dissipation when the body force inputs no energy into the system. Without taking into account the MHD equations, we seek the velocity and magnetic fields that are extremal values of the excess dissipation, with the assumptions that the fields are divergence-free and have fixed values at the boundary. We introduce two Lagrange multipliers $-2 P(x, y, z)$ and $-2 Q(x, y, z)$ to ensure that the fields are divergence-free.
Upon taking the variational derivatives of $\mathcal{D}_{e}-2\langle P \nabla \cdot \mathbf{v}\rangle$ $-2\langle Q \nabla \cdot \mathbf{B}\rangle$ with respect to $\mathbf{v}$ and $\mathbf{B}$ we obtain after a rudimentary calculation

$$
\begin{aligned}
& \nu \nabla^{2} \mathbf{v}+\mathbf{f}-\nabla P=0, \\
& \eta \nabla^{2} \mathbf{B}-\nabla Q=0 .
\end{aligned}
$$

We call these equations, together with the divergence conditions, the Stokes-type equations for MHD.

The two fields are not coupled in these equations, as one expects since $\mathcal{D}_{e}$ is the sum of two functionals that depend separately on the velocity and the magnetic field. Equation (4) for the velocity field is the same as that for Stokes flow, namely, the Navier-Stokes equation for a stationary velocity field in the low Reynolds number limit, where the inertia term and $\mathbf{J} \times \mathbf{B}$ force are neglected. Observe that the Lagrange multiplier, $P$, that was used to enforce $\nabla \cdot \mathbf{v}=0$ turns out to be precisely the pressure. Equation (5) for the magnetic field is more surprising. Here the Lagrange multiplier, $Q$, also appears as a pressure-like term, but it has no trivial physical interpretation. Sometimes the term $\mathbf{B} \cdot \nabla \mathbf{v}$ $-\mathbf{v} \cdot \nabla \mathbf{B}$ of Eq. (2) can be written as a gradient of a scalar function. In which case, the Stokes-type equation for the magnetic field is equivalent to the induction equation for a steady magnetic field. Otherwise, upon choosing $Q=0$, Eq. (5) becomes the low Reynolds number limit of the induction equation for a steady magnetic field.

The above procedure of setting to zero the variational derivatives of $\mathcal{D}_{e}$, subject to the stated conditions, shows that the Stokes-type solution is extremal. However, it remains to demonstrate that this extremal solution actually is a minimum. In fact, it is not difficult to show that the Stokestype solution is minimum over the set of all continuous divergence-free fields with piecewise continuous derivatives that satisfy the line-tied and no-slip boundary conditions. To see this we set $\mathbf{v}=\mathbf{v}_{s}+\mathbf{u}$ and $\mathbf{B}=\mathbf{B}_{s}+\mathbf{b}$, where the $\mathbf{v}_{s}$ and $\mathbf{B}_{s}$ are the Stokes-type solutions of Eqs. (4) and (5). Consequently, $\mathbf{u}$ and $\mathbf{b}$ vanish at the boundary. Inserting these expressions into $\mathcal{D}_{e}$ gives

$$
\begin{aligned}
\mathcal{D}_{e}(\mathbf{v}, \mathbf{B})= & \mathcal{D}_{e}\left(\mathbf{v}_{s}, \mathbf{B}_{s}\right)+\mathcal{D}_{e}(\mathbf{u}, \mathbf{b})+2 \nu\left\langle\nabla \times \mathbf{v}_{s} \cdot \nabla \times \mathbf{v}\right\rangle \\
& +2 \eta\left\langle\nabla \times \mathbf{B}_{s} \cdot \nabla \times \mathbf{b}\right\rangle \\
= & \mathcal{D}_{e}\left(\mathbf{v}_{s}, \mathbf{B}_{s}\right)+\mathcal{D}(\mathbf{u}, \mathbf{b})-2\left(\left\langle\mathbf{u} \cdot\left(\nu \nabla^{2} \mathbf{v}_{s}+\mathbf{f}\right)\right\rangle\right. \\
& \left.+\left\langle\eta \mathbf{b} \cdot \nabla^{2} \mathbf{B}_{s}\right\rangle\right) .
\end{aligned}
$$

Because $\mathbf{v}_{s}$ and $\mathbf{B}_{s}$ solve Eqs. (4) and (5), the term in parentheses vanishes and we are left with

$$
\mathcal{D}_{e}(\mathbf{v}, \mathbf{B})=\mathcal{D}_{e}\left(\mathbf{v}_{s}, \mathbf{B}_{s}\right)+\mathcal{D}(\mathbf{u}, \mathbf{b}) \geqslant \mathcal{D}_{e}\left(\mathbf{v}_{s}, \mathbf{B}_{s}\right),
$$

which establishes the result.

The above result is a straightforward generalization to MHD of analogous results for hydrodynamic flows (see Ref. 29). The calculation is valid for the particular boundary conditions of fixed $\mathbf{v}$ and $\mathbf{B}$ at the boundary, but it is not true for all boundary conditions; for instance, it does not hold if the tangential currents are fixed instead of the magnetic field. For hydrodynamics problems it was proved that if the stress is fixed at the boundary, then Stokes flow minimizes the excess dissipation (defined similarly to $\mathcal{D}_{e}$ with $\mathbf{B} \rightarrow 0$ and taking into account the power input by the stress at the 


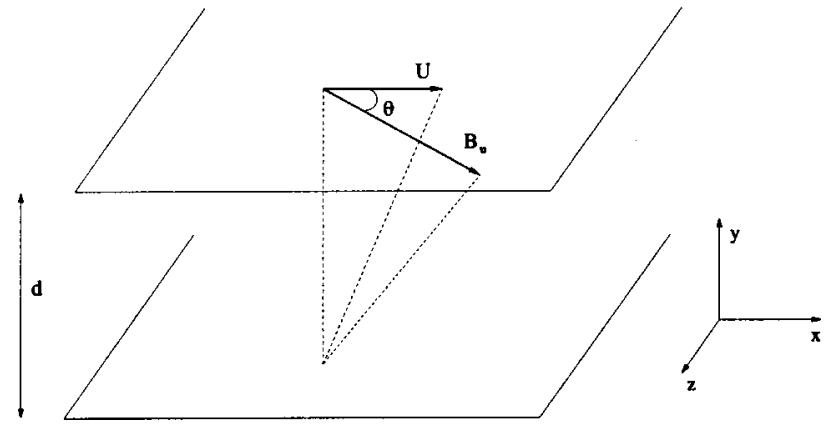

FIG. 1. Sketch of fluid layer with horizontally sheared magnetic and velocity fields.

boundary). This hydrodynamic result can also be easily adapted to MHD for the case of fixed tangential current at the boundary. We do not supply the details here because the resulting lower bound for the dissipation does not provide any information for the sheet pinch model we discuss in Sec. IV.

We return to Eq. (6) in the next section, where we discuss upper bounds on the dissipation rate. However, before doing so we note that another approach is to estimate the lower bound on the excess dissipation rate by using trial functions for $\mathbf{v}$ and $\mathbf{b}$, rather than solving the MHD equations for stationary fields. In Ref. 29 a reciprocal principle was established, whereby the Stokes solution of a hydrodynamic problem can be obtained as the maximizing field of another functional, the maximal value of which is the excess dissipation $\mathcal{D}_{e}$. It appears that the same result can be derived for MHD problems, but we will not pursue this here.

\section{VELOCITY AND MAGNETIC SHEAR LAYER}

We now consider a layer of fluid that can support shear in both the velocity and magnetic fields. We assume a layer of height $d$, where $y$ is the vertical coordinate, with two periodic boundary conditions in the horizontal directions $x$ and $z$, as depicted in Fig. 1. The unit vectors $\mathbf{i}, \mathbf{j}, \mathbf{k}$ are chosen to correspond to the coordinates $(x, y, z)$, respectively. It will turn out that our results are independent of the existence of any uniform horizontal magnetic field, and thus only depend on the shear part of the applied field. We assume the magnetic field is fixed at the upper and lower boundaries. The magnetic shear is imposed by setting $\mathbf{B}(x, d, z)=B_{u} \hat{\rho}$ $=B_{u}(\cos \theta \mathbf{i}+\sin \theta \mathbf{k})$ at the upper surface, $y=d$, and $\mathbf{B}(x, 0, z)=0$ at the bottom surface, $y=0$. Here $B_{u}$ is a constant and $\hat{\rho}$ is a unit vector oriented at the angle $\theta$ with respect to the $x$-axis as depicted in Fig. 1. The velocity shear is induced by moving the upper surface with constant velocity $\mathbf{v}(x, d, z)=U \mathbf{i}$, while keeping the bottom surface immobile. We use the no-slip conditions for the velocity field; i.e., $\mathbf{v}(x, d, z)=U \mathbf{i}$ and $\mathbf{v}(x, 0, z)=0$.

This plane shear configuration can give rise to a variety of shear and tearing instabilities, and indeed has been the subject of extensive linear stability analyses. When the upper boundary is immobile, the configuration is that of the original tearing mode calculations of Ref. 40 (see also Ref. 41), but with the moving upper surface, Kelvin-Helmholtz type instabilities are possible and the tearing mode can be significantly altered. (See, e.g., Ref. 42, where tearing with the inclusion of both the effects of shear flow and viscosity are taken into account.) This configuration can also be viewed as that of plane-Couette flow for a liquid metal subjected to a sheared magnetic field. As noted above, nonlinear stability was addressed in Ref. 32, and a domain in which the basic solution is so-called energy stable was calculated. We revisit this calculation in Sec. III A below, and obtain new results.

Before proceeding to our calculations of Secs. III A and III B that lead to an upper bound on the dissipation rate $\mathcal{D}$, defined by Eq. (3), we make some remarks about $\mathcal{D}$ to set the stage. First, for any quantity $\mathbf{h}$ that is fixed on the boundaries, $\left\langle|\nabla \mathbf{h}|^{2}\right\rangle=\left\langle|\nabla \times \mathbf{h}|^{2}\right\rangle$, where $|\nabla \mathbf{h}|^{2}=\Sigma_{\alpha, \beta}\left(\partial_{\alpha} h_{\beta}\right)^{2}$. Thus,

$$
\mathcal{D}=\nu\left\langle|\nabla \mathbf{v}|^{2}\right\rangle+\eta\left\langle|\nabla \mathbf{B}|^{2}\right\rangle .
$$

The problem at hand has six parameters $B_{u}, U, d, \nu, \eta$, $\theta$, from which we can construct four independent dimensionless numbers. The angle $\theta$ is one of them and various choices can be made for the other three. We choose the magnetic Prandtl number, $P_{m}:=\nu / \eta$, the Reynolds number, Re $:=U d / \nu$, and the equivalent of the Reynolds number constructed with the magnetic field, $M:=B_{u} d / \nu$. By elementary dimensional analysis we see that the dissipation rate has the form

$$
\mathcal{D}=\frac{U^{3}}{d} \phi\left(P_{m}, \operatorname{Re}, M, \theta\right),
$$

where $\phi$ is an unknown function of the dimensionless numbers. Our goal is to find restrictions on the values $\phi$ can obtain.

\section{A. Stokes-type solution and its stability}

At low values of $M$ and Re, one expects the velocity and magnetic fields to be independent. Indeed, a stationary laminar solution of the full set of MHD equations is given by

$$
\mathbf{v}_{s}=\frac{U}{d} y \mathbf{i} \quad \text { and } \quad \mathbf{B}_{s}=\frac{B_{u}}{d} y \hat{\rho} .
$$

For these fields with linear dependence there is no power input by external forces, so the excess dissipation is equal to the dissipation $\mathcal{D}_{l}$. Inserting (9) into (7) gives

$$
\mathcal{D}_{l}=\nu\left(\frac{U}{d}\right)^{2}+\eta\left(\frac{B_{u}}{d}\right)^{2},
$$

which upon comparison with (8) yields

$$
\phi_{l}=\frac{1}{\operatorname{Re}}\left[1+\frac{1}{P_{m}}\left(\frac{M}{R_{e}}\right)^{2}\right] .
$$

Because Eqs. (9) are solutions of the Stokes-type equations (4) and (5), according to the analysis of Sec. II leading to Eq. (6), the laminar dissipation (10) is a lower bound for the total dissipation of any solution of the MHD equations.

If we increase the values of $M$ and $\mathrm{Re}$, we expect the laminar solution to become unstable and possibly turbulent. Using the nonlinear evolution equation for arbitrary perturbations to the laminar solution, we calculate values of $M$ and 
Re below which the integrated sum of the squares of all perturbations decreases to zero. This notion of stability is generally called energy stability. We write $\mathbf{v}=\mathbf{v}_{s}+\mathbf{u}$ and $\mathbf{B}$ $=\mathbf{B}_{s}+\mathbf{b}$, where the perturbations $\mathbf{u}$ and $\mathbf{b}$ have $x, y$, and $z$ components denoted by $\left(u_{1}, u_{2}, u_{3}\right)$ and $\left(b_{1}, b_{2}, b_{3}\right)$, respectively. Note, the perturbations are not assumed to be small as in conventional linear stability analyses. By simple integration by parts, we derive the exact evolution equation for $\mathcal{E}$ $:=\left\langle u^{2}+b^{2}\right\rangle / 2$,

$$
\frac{d \mathcal{E}}{d t}=-\mathcal{Q}(\mathbf{u}, \mathbf{b}),
$$

where the functional

$$
\begin{aligned}
\mathcal{Q}(\mathbf{u}, \mathbf{b}):= & \nu\left\langle|\nabla \mathbf{u}|^{2}\right\rangle+\eta\left\langle|\nabla \mathbf{b}|^{2}\right\rangle+\frac{B_{u}}{d}\left\langle u_{2} \mathbf{b} \cdot \hat{\rho}-b_{2} \mathbf{u} \cdot \hat{\rho}\right\rangle \\
& +\frac{U}{d}\left\langle u_{1} u_{2}-b_{1} b_{2}\right\rangle,
\end{aligned}
$$

is quadratic in both the perturbation velocity and magnetic fields.

The term energy stability is commonly used because $\mathcal{E}$ resembles the spatial average of the perturbation energy. However, the real perturbation energy has additional terms that are linear in the perturbations, which arise because one has expanded about a state that possesses free energy, e.g., in the form of kinetic energy of the equilibrium flow. Such terms can give rise to linear instability or negative energy modes (see, e.g., Ref. 43). Consequently, the real perturbation energy does not provide a norm for stability, and that is why the quantity $\mathcal{E}$ is used. Below we follow the common practice of referring to $\mathcal{E}$ as the perturbation energy.

Observe, Eq. (13) is independent of any horizontal basic magnetic field that is independent of $y$. Consequently, any results obtained concerning energy stability are also independent of such fields. Therefore, the example we are treating is actually somewhat more general than initially supposed. Any horizontal magnetic field applied at the boundaries can be decomposed into its value at the bottom surface plus a shear field, and only the latter part enters into our calculations of energy stability.

We show that up to some critical value of $M$, say $M_{E}$, which is function of $\mathrm{Re}$ and $P_{m}$, the energy of any perturbation to the laminar solution tends to zero. Since this energy is a positive definite quantity, this means that asymptotically the perturbation tends to zero (in $L_{2}$ norm), and for values of $M$ and $\operatorname{Re}$ such that $M$ is smaller than $M_{E}$, the stationary basic state is the only stable solution of the problem.

If $\mathcal{Q}$ is positive for all $\mathbf{u}$ and $\mathbf{b}$, then the basic stationary state is energy stable. We rescale the variables and use $\widetilde{\mathbf{u}}$ $=\lambda \mathbf{u}$ and $\widetilde{\mathbf{b}}=\mu \mathbf{u}$ with $\lambda$ and $\mu$ positive constants. This yields

$$
\begin{aligned}
\mathcal{Q}(\mathbf{u}, \mathbf{b})= & \widetilde{\mathcal{Q}}(\widetilde{\mathbf{u}}, \widetilde{\mathbf{b}}) \\
= & \frac{\nu}{\lambda^{2}}\left\langle|\nabla \widetilde{\mathbf{u}}|^{2}\right\rangle+\frac{\eta}{\mu^{2}}\left\langle|\nabla \widetilde{\mathbf{b}}|^{2}\right\rangle+\frac{B_{u}}{d \mu \lambda}\left\langle\widetilde{u}_{2} \widetilde{\mathbf{b}} \cdot \hat{\rho}-\widetilde{b}_{2} \widetilde{\mathbf{u}} \cdot \hat{\rho}\right\rangle \\
& +\frac{U}{d}\left\langle\frac{1}{\lambda^{2}} \widetilde{u}_{1} \widetilde{u}_{2}-\frac{1}{\mu^{2}} \widetilde{b}_{1} \widetilde{b}_{2}\right\rangle .
\end{aligned}
$$

Clearly, $\mathcal{Q}$ is positive definite if and only if $\widetilde{\mathcal{Q}}$ is positive definite. Henceforth we consider $\widetilde{\mathcal{Q}}$ and drop the tilde to relieve notation. We use the inequalities $-2 u_{1} u_{2} \leqslant u_{1}^{2}+u_{2}^{2}$,

$$
\begin{aligned}
& \left|\left\langle u_{2} \mathbf{b} \cdot \hat{\rho}-b_{2} \mathbf{u} \cdot \hat{\rho}\right\rangle\right| \leqslant \frac{1}{2}\left(\left\langle b^{2}\right\rangle+\left\langle u^{2}\right\rangle\right), \\
& \frac{\pi^{2}}{d^{2}}\left\langle u^{2}\right\rangle \leqslant\left\langle|\nabla \mathbf{u}|^{2}\right\rangle .
\end{aligned}
$$

The last one, which is sometimes called the Poincare inequality, is true for all vector fields periodic in the horizontal coordinates and zero at the top and bottom surfaces (see, for example, Ref. 19). Thus it also holds for the magnetic field and we obtain

$$
\begin{aligned}
\mathcal{Q} \geqslant & \frac{1}{\lambda^{2}}\left(\frac{\nu \pi^{2}}{d^{2}}-\frac{U}{2 d}-\frac{B_{u}}{2 d \alpha}\right)\left\langle u^{2}\right\rangle \\
& +\frac{1}{\mu^{2}}\left(\frac{\eta \pi^{2}}{d^{2}}-\frac{U}{2 d}-\frac{B_{u} \alpha}{2 d}\right)\left\langle b^{2}\right\rangle,
\end{aligned}
$$

where $\alpha=\mu / \lambda>0$. Note, because $\theta$ does not enter into this lower bound on $\mathcal{Q}$, our energy stability domain for the laminar solution will be independent of $\theta$.

A sufficient condition for $\mathcal{Q}$ to be positive definite is that $2 \pi^{2}-(\operatorname{Re}+M / \alpha)>0$ and $2 \pi^{2}-P_{m}(\operatorname{Re}+M \alpha)>0$. Because $\alpha>0$ is arbitrary, we can choose it in an optimum way to obtain a maximal value of $M$, below which both inequalities are satisfied. This gives

$$
M_{E}^{2}=\left(2 \pi^{2}-\operatorname{Re}\right)\left(2 \pi^{2} / P_{m}-\operatorname{Re}\right),
$$

where, if $M \leqslant M_{E}$ the laminar flow is energy stable. Technically this follows because $d \mathcal{E} / d t=-\mathcal{Q} \leqslant-C_{B} \mathcal{E}$, where $C_{B}$ $>0$ is the smaller of the two coefficients of (17), can be integrated by Gronwall's lemma to obtain $\mathcal{E}(t) \leqslant \mathcal{E}(0)$ $\times \exp \left(-C_{B} t\right)$.

In Ref. 32 it was shown that $\operatorname{Re} \max \left(1, P_{m}\right)$ $+M_{E} \max \left(1, P_{m}\right)=2 \pi^{2}$ defines an energy stability boundary. We plot this result along with our results in Fig. 2, which depicts stability domains for different values of $P_{m}$ in the $\left[\operatorname{Re} \max \left(1, P_{m}\right), M_{E} \max \left(1, P_{m}\right)\right]$ plane. With this choice of variables, the result of Ref. 32 is independent of $P_{m}$, and the domains described by Eq. (18) are the same for $P_{m}$ and $P_{m}^{-1}$. Therefore it is only necessary to plot the $P_{m} \geqslant 1$ case. For $P_{m}=1$ our result is the same as that of Ref. 32, but for $P_{m} \neq 1$ our domains are larger. This enlargement of the stability domain came from the variable change in Eq. (14) that led to a better estimate of $\mathcal{Q}$. Note that this in essence follows from the general inequality $2 a b \leqslant \alpha a^{2}+b^{2} / \alpha$, which is true for any real $a$ and $b$ and $\alpha$ strictly positive.

For $\mathrm{Re}=0$, Eq. (18) reduces to $M_{E}=2 \pi^{2} \sqrt{P_{m}}$. If we use the dimensionless parameter $M^{\prime}=B d / \sqrt{\nu \eta}$ instead of $M$, then the abscissa boundary of the stability domain is independent of the magnetic Prandtl number, just as the critical Rayleigh number is independent of the thermal Prandtl number in thermal convection of ordinary fluids. For $M=0$, we recover the result for plane-Couette instability. Introducing the magnetic Reynolds number $R_{m}=\operatorname{Re} P_{m}$, the abscissa boundary of the domain is given by $\max \left(\operatorname{Re}, R_{m}\right) \leqslant 2 \pi^{2}$. For $\nu \leqslant \eta, \operatorname{Re} \geqslant R_{m}$, and the domain is the same as that for an 


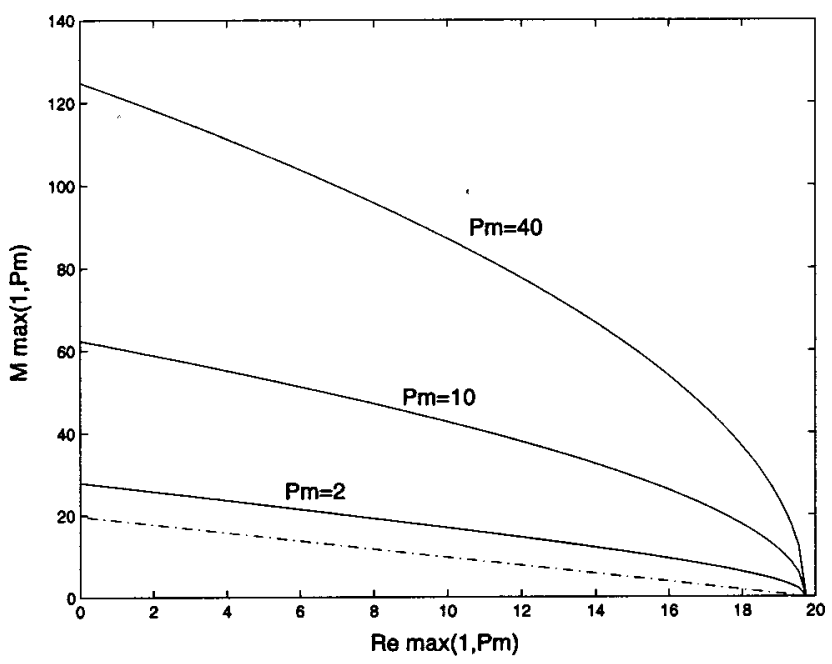

FIG. 2. Boundary of domains in which the basic state is energy stable for a shear layer with both velocity and magnetic shear in the $\left[\right.$ Re $\left.\max \left(1, P_{m}\right), M \max \left(1, P_{m}\right)\right]$ plane. Below a curve, the laminar stationary solution is energy stable for the particular value of $P_{m}$. The dashed line is the result from Ref. 32, which is equal to our result for $P_{m}=1$. The continuous lines are our results for $P_{m}>1$. These curves are the same for $P_{m}$ and $P_{m}^{-1}$

ordinary fluid. For $\nu \geqslant \eta, \operatorname{Re} \leqslant R_{m}$, the boundary of the domain corresponds to a lower value of Re than that for an ordinary fluid.

Although it is known from linear stability analyses that velocity shear can either stabilize or destabilize MHD equilibrium configurations (see, e.g., Ref. 44), a physical explanation for the stability boundaries is not forthcoming and this points to the limitation of energy stability arguments. Nonlinear energy arguments, unlike linear stability analysis, have no eigenfunctions and eigenvalues, and one cannot always be sure of what causes the instability. Results may be due either to calculational limitations or to the physics. For example, in the present situation, a larger stability domain could be achieved by searching for the maximal value of $M$ and Re such that $\mathcal{Q}$ is positive definite. These maximal values define the energy stability domain in the $(M, \operatorname{Re})$ plane analogous to the critical energy Reynolds number obtained for hydrodynamic instabilities in Refs. 28 and 31. Because our estimates above are not sharp, the values of $M$ and Re we obtain are inside this maximal energy stability domain. Note that in the case $M=0$, this maximal domain can be easily computed from (13) because the quadratic form is then the sum of two quadratic forms depending separately on $\mathbf{u}$ and $\mathbf{b}$. The maximal domain for energy stability can be shown to satisfy $\max \left(\operatorname{Re}, R_{m}\right) \leqslant 82.1 \ldots$. Thus, for $M=0$ the sharp result and the maximal domain are the same up to a numerical factor and have the same dependence on $P_{m}$. It is also possible that the stability domain might be enlarged by incorporating other ideal MHD invariants into the calculation, which could help to identify a different positive definite quantity besides $\mathcal{E}$. It was for stability reasons like this that Kruskal, Oberman, Gardner, and others sought invariants in the early days of plasma physics (ca. 1960), and we will never be sure whether or not we have them all.

\section{B. Upper bounds for the dissipation}

Outside the maximal domain in which the basic solution is energy stable, the flow can be nonstationary and possibly turbulent. It is in this region that the background method can be used to find an upper bound on the dissipation rate. We now describe this method in the context of the shear layer system at hand.

Like before, we decompose the velocity and magnetic fields into two parts: $\mathbf{v}=\mathbf{v}_{b}+\mathbf{u}$ and $\mathbf{B}=\mathbf{B}_{b}+\mathbf{b}$, where $\mathbf{v}_{b}$ and $\mathbf{B}_{b}$ are now background fields, which unlike before need not be solutions and can be chosen for convenience. We suppose the background fields satisfy the boundary conditions and depend only on $y$, such that $\mathbf{v}_{b}=U_{b}(y) \mathbf{i}$, with $U_{b}(0)=0$ and $U_{b}(d)=U$, and $\mathbf{B}_{b}=B_{b}(y) \hat{\rho}$, with $B_{b}(0)=0$ and $B_{b}(d)$ $=B_{u}$.

Given this decomposition, Eq. (7) for the spatial average of the dissipation rate can be written as follows:

$\mathcal{D}=\nu\left\langle|\nabla \mathbf{u}|^{2}+2 U_{b}^{\prime} u_{1}^{\prime}+U_{b}^{\prime 2}\right\rangle+\eta\left\langle|\nabla \mathbf{b}|^{2}+2 \mathbf{B}_{b}^{\prime} \cdot \mathbf{b}^{\prime}+B_{b}^{\prime 2}\right\rangle$,

where prime denotes $\partial / \partial y$. Adding $\mathcal{D} / 2$ to the evolution equation for $\mathcal{E}=\left\langle u^{2}+b^{2}\right\rangle / 2$ yields

$$
\frac{d \mathcal{E}}{d t}+\frac{\mathcal{D}}{2}=\frac{\mathcal{F}}{2}-\mathcal{P}
$$

where $\mathcal{F}\left(B_{b}, U_{b}\right)$ is a functional that depends only the background fields and $\mathcal{P}\left(\mathbf{u}, \mathbf{b}, B_{b}, U_{b}\right)$ is a functional that is quadratic in the perturbation fields. These functionals are defined as follows:

$$
\begin{aligned}
& \mathcal{F}\left(B_{b}, U_{b}\right)=\nu\left\langle U_{b}^{\prime 2}\right\rangle+\eta\left\langle B_{b}^{\prime 2}\right\rangle, \\
& \mathcal{P}\left(\mathbf{u}, \mathbf{b}, B_{b}, U_{b}\right)= \frac{\nu}{2}\left\langle|\nabla \mathbf{u}|^{2}\right\rangle+\frac{\eta}{2}\left\langle|\nabla \mathbf{b}|^{2}\right\rangle+\left\langle u_{2} \mathbf{b} \cdot \mathbf{B}_{b}^{\prime}\right. \\
&\left.-b_{2} \mathbf{u} \cdot \mathbf{B}_{b}^{\prime}\right\rangle+\left\langle U_{b}^{\prime}\left(u_{1} u_{2}-b_{1} b_{2}\right)\right\rangle .
\end{aligned}
$$

Now, if we time average (20) so that $d \mathcal{E} / d t$ drops out and if we find background fields such that $\mathcal{P}$ is positive definite, then we obtain an upper bound for $\overline{\mathcal{D}}$, the time average of the dissipation rate,

$$
\overline{\mathcal{D}} \leqslant \mathcal{F}\left(B_{b}, U_{b}\right) .
$$

Note that adding $\mathcal{D} / 2$ removed terms linear in $\mathbf{u}$ and $\mathbf{b}$ that arise from the dissipation terms of (1) and (2). This is a key point, because in removing these terms one is left with the form $\mathcal{F} / 2-\mathcal{P}$, where $\mathcal{P}$ being quadratic is easily bounded.

For the magnetic (respectively, velocity) background field, we use a piecewise linear function equal to $B_{u} / 2$ (respectively, $U / 2$ ) for $\delta_{b} \leqslant y \leqslant d-\delta_{b}$ (respectively, $\delta_{u} \leqslant y \leqslant d$ $\left.-\delta_{u}\right)$ and of slope $B_{u} /\left(2 \delta_{b}\right)$ [respectively, $U /\left(2 \delta_{u}\right)$ ] for the two boundary layers, as sketched in Fig. 3. Note that both boundary layer thicknesses, $\delta_{u}$ and $\delta_{b}$, must each be smaller than $d / 2$.

Upon integration by parts and making use of Hölder's inequality, it is easy to show that 


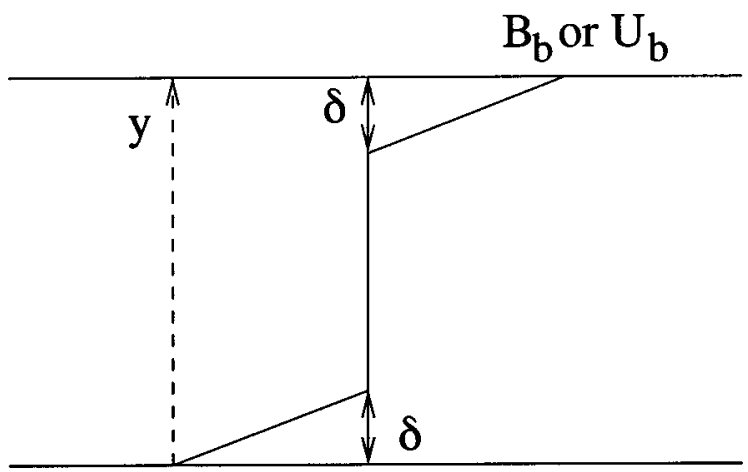

FIG. 3. Piecewise linear background field. The thickness of the boundary layer is $\delta$ ( $\delta_{b}$ for the magnetic field and $\delta_{u}$ for the velocity field).

$\left|\left\langle B_{b}^{\prime}\left(b_{2} \mathbf{u} \cdot \hat{\rho}-u_{2} \mathbf{b} \cdot \hat{\rho}\right)\right\rangle\right| \leqslant \frac{B_{u} \delta_{b}}{8}\left\langle\alpha|\nabla \mathbf{u}|^{2}+\frac{1}{\alpha}|\nabla \mathbf{b}|^{2}\right\rangle$,

$\left|\left\langle U_{b}^{\prime}\left(u_{1} u_{2}-b_{1} b_{2}\right)\right\rangle\right| \leqslant \frac{U \delta_{u}}{8}\left\langle|\nabla \mathbf{u}|^{2}+|\nabla \mathbf{b}|^{2}\right\rangle$

(see, e.g., Ref. 19), and hence show that

$$
\begin{aligned}
\mathcal{P} \geqslant & \left(\frac{\nu}{2}-\frac{U \delta_{u}}{8}-\frac{B_{u} \delta_{b} \alpha}{8}\right)\left\langle|\nabla \mathbf{u}|^{2}\right\rangle+\left(\frac{\eta}{2}-\frac{U \delta_{u}}{8}-\frac{B_{u} \delta_{b}}{8 \alpha}\right) \\
& \times\left\langle|\nabla \mathbf{b}|^{2}\right\rangle,
\end{aligned}
$$

where, as in Sec. III A, $\alpha>0$ is arbitrary. Note that $\theta$ does not enter into this bound for $\mathcal{P}$ and, consequently, the upper bound for the dissipation will also be independent of it.

We first choose the thickness of the boundary layers so that $\mathcal{P} \geqslant 0$ and then we minimize (21) evaluated on our choice of background fields,

$$
\mathcal{F}=\frac{1}{2 d}\left(\frac{\nu U^{2}}{\delta_{u}}+\frac{\eta B_{u}^{2}}{\delta_{b}}\right),
$$

with the constraints that $\delta_{u}$ and $\delta_{b}$ are each smaller than $d / 2$. If $\delta_{u}$ is fixed, the minimum of $\mathcal{F}$ is obtained for the highest possible $\delta_{b}$. Introducing $\xi:=U \delta_{u} / \nu$, we calculate from the coefficients of (24) the maximum $\delta_{b}$ that ensures positivity of $\mathcal{P}$. Following the same procedure that let to (18), we obtain

$$
\frac{B_{u} \delta_{b}}{\nu}=g(\xi)=\sqrt{(4-\xi)\left(4 / P_{m}-\xi\right)},
$$

where $\xi \leqslant \min \left(4,4 / P_{m}\right)$. Now let $\mathcal{F}_{\xi}$ be the upper bound of $\overline{\mathcal{D}}$ corresponding to a given value of the parameter $\xi$; i.e.,

$$
\mathcal{F}_{\xi}=\frac{U^{3}}{2 d}\left(\frac{1}{\xi}+\frac{C}{g(\xi)}\right)
$$

where $C:=\eta B_{u}^{3} /\left(\nu U^{3}\right)$. Minimizing $\mathcal{F}_{\xi}$ over $\xi$, we find that the minimum is achieved for $\xi$ equal to $\xi_{0}$, the positive solution of

$$
\frac{\left[(4-\xi)\left(4 / P_{m}-\xi\right)\right]^{3 / 2}}{\xi^{2}}=C\left(2+2 / P_{m}-\xi\right) .
$$

In terms of $\xi$, the two constraints on the thickness of the boundary layers are $\xi \leqslant \operatorname{Re} / 2$ and $g(\xi) \leqslant M / 2$. If $\xi_{0} \geqslant \operatorname{Re} / 2$,

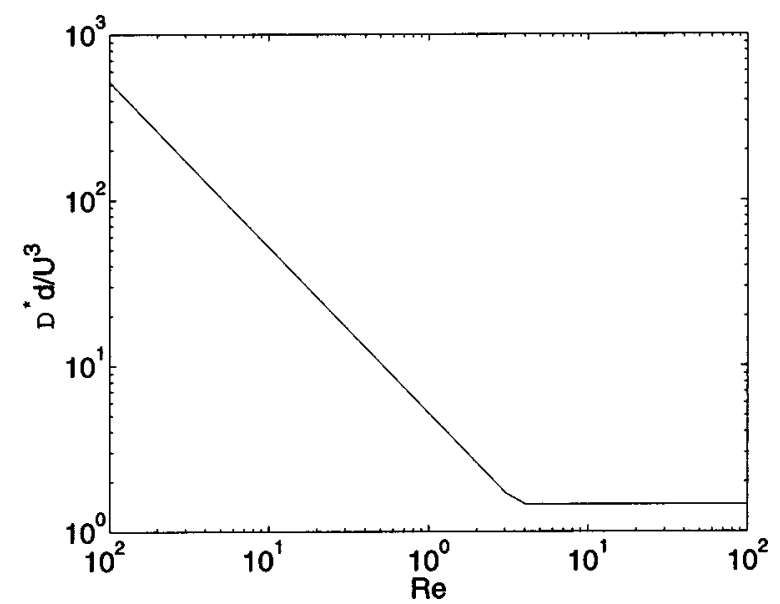

FIG. 4. Value of the upper bound for the dissipation $\mathcal{D}^{*} d / U^{3}$ as a function of Re for $P_{m}=1$ and $C:=\eta B_{u}^{3} /\left(\nu U^{3}\right)=2$.

then the boundary layer for the velocity is $d / 2$ and $\xi$ $=\operatorname{Re} / 2$. If $g(\xi) \geqslant M / 2$, then the thickness of the magnetic boundary layer is $d / 2$ and $g(\xi)=M / 2$. Such values of the thicknesses of the boundary layers are obtained for low values of Re and $M$. In which case, the dissipation is that of the laminar solution. For higher values of Re and $M$, the upper bound is obtained for (27) evaluated at $\xi=\xi_{0}$, which requires numerical evaluation.

In Fig. 4 we plot $\mathcal{D}^{*} d / U^{3}$, where $\mathcal{D}^{*}$ is the upper bound for the dissipation, as a function of $\operatorname{Re}$ for $C=2$ and $P_{m}$ $=1$. At low values of $\operatorname{Re}, \mathcal{D}^{*} d / U^{3}$ is decreasing. This behavior occurs when the dissipation is equal to the laminar dissipation rate and the thickness of the boundary layers are $d / 2$. For high $\operatorname{Re}, \mathcal{D}^{*} d / U^{3}$ approaches an asymptote that depends on $C$ and $P_{m}$. We plot the high Re value of $\mathcal{D}^{*} d / U^{3}$ as a function of $P_{m}$ for different values of $C$ in Fig. 5. When $R_{e}$ is large $\xi$ is obtained from Eq. (28), which can be expanded along with (26) in the limits of small and large $P_{m}$ and used with (27) to obtain the asymptotic behaviors

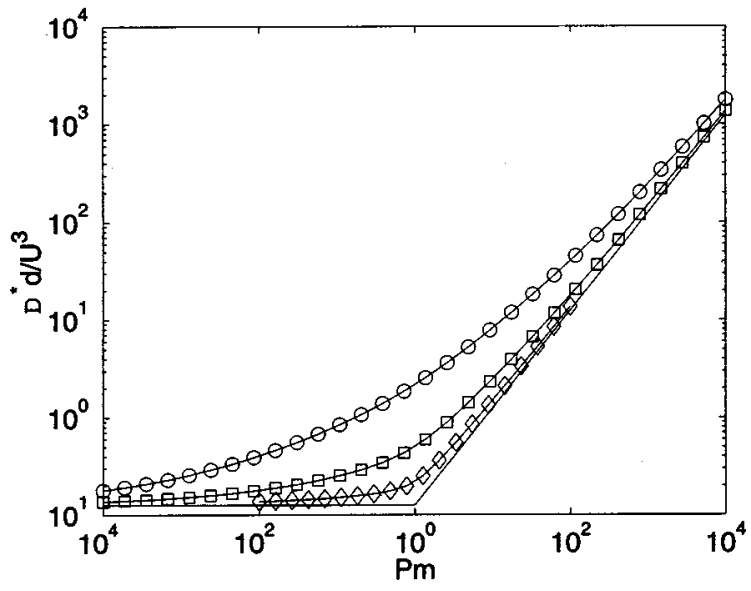

FIG. 5. Value of the upper bound for the dissipation $\mathcal{D}^{*} d / U^{3}$ for infinite Re as a function of $P_{m}$ for $C=10(\bigcirc), C=1(\square)$, and $C=0.1(\diamond)$. The continuous line depicts the asymptotic behavior of (29). 


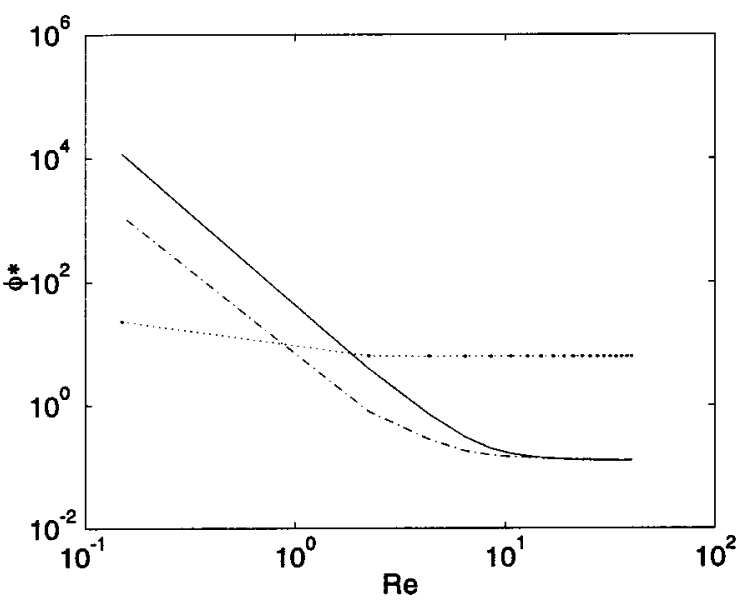

FIG. 6. Value of the upper bound for the dissipation $\phi^{*}$ as a function of $\operatorname{Re}$ for $M=2$ and $P_{m}=0.1(-), P_{m}=1(-\cdot)$, and $P_{m}=50(\cdot)$.

$$
\lim _{P_{m} \rightarrow 0}\left(\lim _{\operatorname{Re} \rightarrow \infty} \frac{\mathcal{D}^{*} d}{U^{3}}\right)=\frac{1}{8} \text { and } \lim _{P_{m} \rightarrow \infty}\left(\lim _{\operatorname{Re} \rightarrow \infty} \frac{\mathcal{D}^{*} d}{U^{3}}\right) \simeq \frac{P_{m}}{8}
$$

which are plotted as a continuous line in Fig. 5.

In terms of the nondimensional dissipation $\mathcal{D}^{*} d / U^{3}$ $=\phi^{*}\left(P_{m}, R_{e}, M\right)$. If we fix $M$ and $P_{m}, \phi^{*}$ decreases for low values of Re and tends to a constant at high Re. We plot $\phi^{*}$ as a function of $\operatorname{Re}$ for $M=2$ and different values of $P_{m}$ in Fig. 6. The asymptotic behavior of $\phi^{*}$ at fixed $M$ is $\lim _{\operatorname{Re} \rightarrow \infty} \phi^{*}=\max \left(1, P_{m}\right) / 8$. If we fix Re and $P_{m}$ and vary $M$, $\phi^{*}$ decreases and tends to a constant. The asymptotic behavior of $\mathcal{D}^{*}$ is then $\mathcal{D}^{*} \simeq B^{3} /\left(8 d P_{m}^{1 / 2}\right)$ for $M \rightarrow \infty$.

The dependence of $\phi^{*}$ with $P_{m}$ for infinite Re and fixed $M$ is similar to that of the boundary of the domain in which the basic state is energy stable. Here again a possible explanation of this scaling is that the bound does not depend on any uniform horizontal magnetic field. Therefore, if such a field is applied, the total dissipation can increase when $\eta$ decreases because there is more Ohmic dissipation. However, this linear increase with $P_{m}$ differs from what is expected based on heuristic turbulence ideas. For example, in the early work of Ref. 45 it was assumed that the magnetic and kinetic energies come into equipartition and that energy is dissipated by viscosity and resistivity at an equal rate. This idea causes $\nu$ and $\eta$ to enter into various formulas in a symmetric way (e.g., the Kolmogoroff scale is determined by a modified Reynolds number with $\nu$ replaced by $\nu+\eta)$, and with this line of argument one expects the dissipation to approach a finite value when both diffusion coefficients $\nu$ and $\eta$ tend to zero. It is possible to obtain a scaling law where the Ohmic dissipation dominates the viscous dissipation (see, for example, Ref. 46), but whether or not this is a physical effect remains to be determined.

\section{PLANE SHEET PINCH}

Next we study a model of a sheet pinch composed of an MHD fluid located between two horizontal surfaces separated by a distance $d$. This problem has the same geometry as that of the shear layer of Sec. III, but different boundary conditions are adopted. We assume no-slip and that both of the bounding surfaces are at rest; i.e. $\mathbf{v}=0$ at $y=0$ and $y$ $=d$. The same notation as shown in Fig. 1 is used. (Note, the same bound we obtain can be derived with the stress-free conditions, $\partial v_{x} / \partial y=\partial v_{z} / \partial y=0$ at $y=0$ and $y=d$.) In addition, we assume that the normal component of the magnetic field, $B_{y}$, vanishes at both the upper and lower surfaces, and that the horizontal current is fixed to be $J_{0} \mathbf{k}$ at both surfaces. In terms of the magnetic field this condition gives $\partial B_{x} / \partial y=J_{0}$ and $\partial B_{z} / \partial y=0$.

An equilibrium solution that satisfies these boundary conditions is $\mathbf{v}_{0}=0, \mathbf{B}_{0}=\left(J_{0} y+C_{1}\right) \mathbf{i}+C_{2} \mathbf{k}$, where $C_{1}$ and $C_{2}$ are constants. The dissipation rate, $\mathcal{D}=\nu\left\langle|\nabla \mathbf{v}|^{2}\right\rangle$ $+\eta\left\langle|\nabla \mathbf{B}|^{2}\right\rangle$, evaluated on this solution is $\mathcal{D}_{0}=\eta J_{0}^{2}$. Because the result of Eq. (6) for $\mathcal{D}_{e}$ was obtained with different boundary conditions, we cannot assume that $\mathcal{D}_{0}$ is a minimum for the dissipation rate. Moreover, the evolution equation for $\mathcal{E}$ is independent of any horizontal uniform magnetic field, and so we can set $C_{1}=C_{2}=0$.

Again we write $\mathbf{B}=\mathbf{B}_{b}+\mathbf{b}$, where the background field is chosen to be of the form $\mathbf{B}_{b}=B_{1}(y) \mathbf{i}$, with the boundary conditions $B_{1}^{\prime}(0)=B_{1}^{\prime}(d)=J_{0}$. For the present problem, we do not expand the velocity field about a background. The equation for $\mathcal{E}=\left\langle v^{2}+b^{2}\right\rangle / 2$ is now

$$
\frac{d \mathcal{E}}{d t}+\frac{\mathcal{D}}{2}=\frac{\mathcal{G}}{2}-\mathcal{R}
$$

with

$\mathcal{G}\left(B_{1}\right)=\eta\left\langle B_{1}^{\prime 2}\right\rangle$,

$$
\begin{aligned}
\mathcal{R}\left(\mathbf{v}, \mathbf{b}, B_{b}\right)= & \frac{\nu}{2}\left\langle|\nabla \mathbf{v}|^{2}\right\rangle+\frac{\eta}{2}\left\langle|\nabla \mathbf{b}|^{2}\right\rangle+\left\langle B_{1}^{\prime}\left(v_{2} b_{1}-v_{1} b_{2}\right)\right\rangle \\
& -\frac{\eta J_{0}}{V} \int_{S} b_{1} d x d z,
\end{aligned}
$$

where in the last term of (31) $S$ denotes the bounding surfaces located at $y=0$ and $y=d$. This surface contribution, which arises because of the boundary conditions of this section, can be changed into a volume integral by using $\int_{S} b_{1} d x d z / V=\left\langle\partial b_{1} / \partial y\right\rangle$. To bound this surface term, we observe

$$
|\nabla \mathbf{b}|^{2}-2 J_{0} \frac{\partial b_{1}}{\partial y} \geqslant-J_{0}^{2}
$$

where we have used $|\nabla \mathbf{b}|^{2} \geqslant\left(\partial b_{1} / \partial y\right)^{2}$. We use a piecewise linear profile for $B_{1}^{\prime}$ of the form $B_{1}^{\prime}(y)=0$ if $\delta \leqslant y \leqslant d-\delta$, $B_{1}^{\prime}(y)=-J_{0}(y-\delta) / \delta$ if $y \leqslant \delta$, and $B_{1}^{\prime}(y)=J_{0}(y+\delta-d) / \delta$ if $y \geqslant d-\delta$. The expression for $B_{1}$ can be deduced by trivial integrations, but it does not enter into the upper bound result. We get $\left\langle B_{1}^{\prime 2}\right\rangle=2 J_{0} \delta /(3 d)$. We then use two results: $\lim _{\delta \rightarrow 0}\left\langle B_{1}^{\prime 2}\right\rangle=0$ and $\lim _{\delta \rightarrow 0} \mathcal{R}\left(\mathbf{v}, \mathbf{b}, B_{b}\right) \geqslant-\eta J_{0}^{2} / 2$. Therefore, the limit $\delta \rightarrow 0$ of Eq. (30) gives

$$
\frac{d \mathcal{E}}{d t}+\frac{\mathcal{D}}{2} \leqslant J_{0}^{2}
$$


Thus upon time averaging as in Sec. III B, we obtain the following upper bound for the time average of the dissipation: $\mathcal{D}^{*}=\eta J_{0}^{2}$.

Note, had we chosen the stress-free and no penetration boundary conditions for the velocity field, we obtain exactly the same results because Eq. (30) holds also in this case.

Our conclusion is that the upper bound for the dissipation is equal to the dissipation for the steady solution. A first consequence is that this bound cannot be improved if the manifold over which we optimize $\mathcal{D}$ contains the steady solution. Another consequence is that the dissipation tends to zero with $\eta$. As described by Paparella et al. in Ref. 35, this is an antiturbulence theorem. The physical behavior of our pinch system and that of Ref. 35 are different. Whereas their simulations show instabilities of the laminar flow and formation of boundary layers, the numerical simulations of the voltage-driven sheet pinch of Ref. 37 show that the static solution is always stable, which is consistent with our upper bound result. This is also consistent with linear tearing mode theory because the static solution in this case is one of constant current across the channel, and consequently the tearing mode drive $\Delta^{\prime}$ (see, e.g., Ref. 41), which measures the singular tearing layer current, vanishes.

\section{CONCLUSION}

We have reported various results on bounds on the dissipation in MHD flows. We defined the Stokes-type solution of the MHD problem and have shown that it minimizes the dissipation over any divergence-free velocity and magnetic fields that are fixed at the boundary. Using the background method, we calculated upper bounds for the dissipation in two examples of MHD flows. In the case of a shear layer with both velocity and magnetic shear, an upper bound was derived and its dependence on the parameters was discussed. As a by-product of this calculation, an improved energy stability domain was obtained. In the case of the sheet pinch, the upper bound for the dissipation was achieved by the equilibrium solution, and it was shown to tend to zero with the resistivity. This is an antiturbulence theorem in the sense that there is no residual dissipation in the limit of zero diffusion coefficient.

Note that the two examples differ only by the boundary conditions, which determine the manner in which energy is put into the system, but their physical behavior is completely different. In our companion paper (Ref. 27) we explore Hartmann flow in a channel, where a magnetic field perpendicular to the boundary is imposed. There we are able to compare our calculations with data from several experiments.

The techniques developed in this paper can be implemented for other geometries. Using the boundary conditions of Sec. IV, we have studied the behavior of the dissipation for a periodic cylindrical configuration, a configuration that more closely mimics toroidal confinement devices. Although this calculation is technically more complicated, the results are qualitatively the same; i.e. there is no residual dissipation when the diffusion coefficients tend to zero. In the future we hope to explore additional boundary conditions and configurations. Of particular interest would be to explore realistic boundary conditions that describe nonideal conductors and insulators. Also, our estimates of the quadratic forms in the case of the shear layer where rough. The domain in which the basic state is energy stable and the upper bound for the dissipation can certainly be improved by using more accurate inequalities. And, as noted before, they may also be improved by taking into account the dissipative dynamics of other ideal invariants, such as the magnetic helicity or the cross helicity.

\section{ACKNOWLEDGMENTS}

We would all like to acknowledge helpful discussions with J. Keller and R. Kerswell. P.J.M. gladly thanks A. Aydemir and P. Watson for several informative conversations.

The hospitality and support of the 2002 GFD Summer Program at the Woods Hole Oceanographic Institution are gladly acknowledged. The GFD program is supported by the US NSF Contract No. OCE-98-10647 and ONR Contract No. N00014-97-1-0934. P.J.M. acknowledges support by the US DOE Contract No. DE-FG03-96ER-54346. C.R.D. was supported in part by US NSF Award PHY-9900635.

${ }^{1}$ W. D. McComb, The Physics of Fluid Turbulence (Oxford University Press, Oxford, 1990).

${ }^{2}$ J. A. Krommes, Phys. Rep. 360, 1 (2002).

${ }^{3}$ A. Sommerfeld, Mechanics of Deformable Bodies: Lectures on Theoretical Physics Volume II (Academic, New York, 1964).

${ }^{4}$ E. N. Lorenz, J. Fluid Mech. 55, 545 (1972).

${ }^{5}$ R. H. Kraichnan, in Theoretical Approaches to Turbulence, Vol. 58 of Applied Mathematical Sciences Series, edited by D. L. Dwoyer, M. Y. Hussaini, and R. G. Voigt (Springer, New York, 1985), Chap. V, p. 91.

${ }^{6}$ T. Williams, E. R. Tracy, and G. Vahala, Phys. Rev. Lett. 59, 1922 (1987).

${ }^{7}$ R. H. Kraichnan and S. Chen, Physica D 37, 160 (1989).

${ }^{8}$ Z.-S. She and E. Jackson, Phys. Rev. Lett. 70, 1255 (1993).

${ }^{9}$ J. C. Bowman and J. A. Krommes, Phys. Plasmas 4, 3895 (1997).

${ }^{10}$ J. C. Bowman, B. A. Shadwick, and P. J. Morrison, Phys. Rev. Lett. 83, 5491 (1999).

${ }^{11}$ W. V. R. Malkus, Proc. R. Soc. London, Ser. A 225, 196 (1954).

${ }^{12}$ L. N. Howard, J. Fluid Mech. 17, 405 (1963).

${ }^{13}$ F. H. Busse, J. Fluid Mech. 41, 219 (1970).

${ }^{14}$ A. Soward, Geophys. Astrophys. Fluid Dyn. 15, 317 (1980).

${ }^{15}$ C.-B. Kim and J. Krommes, Phys. Rev. A 42, 7487 (1990).

${ }^{16}$ C. Y. Wang, A. Bhattacharjee, and E. Hameiri, Physica B 3, 715 (1991).

${ }^{17}$ C. Y. Wang and A. Bhattacharjee, Phys. Fluids B 3, 3462 (1991).

${ }^{18}$ F. H. Busse, "Upper bounds on the resistivity of a turbulent current layer," preprint (2003).

${ }^{19}$ C. R. Doering and P. Constantin, Phys. Rev. Lett. 69, 1648 (1992).

${ }^{20} \mathrm{E}$. Hopf, Lecture Series of the Symposium on Partial Differential Equations, Berkeley, 1955 (University of Kansas Press, Lawerence, 1957).

${ }^{21}$ T. K. Fowler, "Thermodynamics of unstable plasmas," in Advances in Plasma Physics, edited by A. Simon and W. B. Thompson (Wiley, New York, 1968), Vol. 1, p. 201.

${ }^{22}$ A. Brizard, T. K. Fowler, D. Hua, and P. J. Morrison, Comments Plasma Phys. Controlled Fusion 14, 263 (1991).

${ }^{23}$ C. R. Doering and P. Constantin, Phys. Rev. E 49, 4087 (1994).

${ }^{24}$ P. Constantin and C. R. Doering, Phys. Rev. E 51, 3192 (1995).

${ }^{25}$ C. R. Doering and P. Constantin, Phys. Rev. E 53, 5957 (1996).

${ }^{26}$ C. R. Doering, E. A. Spiegel, and R. A. Worthing, Phys. Fluids 12, 1955 (2000).

${ }^{27}$ A. Alexakis, F. Pétrélis, P. J. Morrison, and C. R. Doering, Phys. Plasmas 10, 4324 (2003)

${ }^{28}$ J. Serrin, in Handbuch der Physik, edited by S. Flügge (Springer, Berlin, 1959), Vol. VIII, Pt. 1, p. 124

${ }^{29}$ J. B. Keller, A. Rubenfeld, and J. E. Molyneux, J. Fluid Mech. 30, 97 (1967).

${ }^{30}$ D. Montgomery, in Trends in Theoretical Physics, edited by P. J. Ellis and Y. C. Tang (Addison-Wesley, New York, 1989), Vol. I, pp. 239-262. 
${ }^{31}$ B. Straughan, The Energy Method, Stability, and Nonlinear Convection (Springer-Verlag, New York, 1991).

${ }^{32}$ H. Tasso and S. J. Camargo, Nuovo Cimento 107B, 733 (1992).

${ }^{33}$ L. Ofman, P. J. Morrison, and R. Steinolfson, Astrophys. J. 417, 748 (1993).

${ }^{34}$ H. Tasso, Nuovo Cimento 108B, 827 (1993).

${ }^{35}$ F. Paparella and W. R. Young, J. Fluid Mech. 466, 205 (2002).

${ }^{36}$ J. Siggers (private communication).

${ }^{37}$ N. Seehafer, E. Zienicke, and F. Feudel, Phys. Rev. E 54, 2863 (1996).

${ }^{38}$ X. Shan, D. Montgomery, and H. Chen, Phys. Rev. A 44, 6800 (1991).

${ }^{39}$ J. Killeen, "Computational problems in plasma physics and controlled thermonuclear research," in Physics of Hot Plasmas: Scottish Universities
Summer School 1968, edited by B. J. Rye and J. C. Taylor (Plenum, New York, 1970), pp. 202-255.

${ }^{40}$ H. P. Furth, J. Killeen, and M. N. Rosenbluth, Phys. Fluids 6, 459 (1963).

${ }^{41} \mathrm{G}$. Bateman, MHD Instabibities (MIT Press, Cambridge, MA, 1978).

${ }^{42}$ X. L. Chen and P. J. Morrison, Phys. Fluids B 2, 2575 (1990).

${ }^{43}$ P. J. Morrison, Rev. Mod. Phys. 70, 467 (1998).

${ }^{44}$ X. L. Chen and P. J. Morrison, Phys. Fluids B 3, 863 (1991).

${ }^{45}$ T. D. Lee, Q. Appl. Math. 10, 69 (1952).

${ }^{46} \mathrm{R}$. Moreau and A. Alemany, "MHD homogeneous turbulence for small magnetic Reynolds number," in MHD-Flows and Turbulence, edited by $\mathrm{H}$. Branover (Wiley, New York, 1976), pp. 51-71. 